\title{
Phenotype-Genotype Correlation in Children with Neurofibromatosis Type 1
}

\author{
Christophe Barrea ${ }^{1}$ Sandrine Vaessen ${ }^{1}$ Saskia Bulk ${ }^{2} \quad$ Julie Harvengt $^{2}$ Jean-Paul Misson ${ }^{1}$ \\ 1 Department of Pediatrics, Centre Regional de la Citadelle, Liege, Belgium \\ ${ }^{2}$ Department of Genetics, Centre Hospitalier Universitaire, Liege, Belgium \\ Neuropediatrics \\ Address for correspondence Christophe Barrea, MD, Department of \\ Pediatrics, Centre Hospitalier Régional de la Citadelle de Liège, \\ Boulevard du Douzième de Ligne 14000 Liège, Belgium \\ (e-mail: chbarrea@gmail.com).
}

\author{
Abstract \\ Keywords \\ - phenotype \\ - neurofibromatosis \\ - genetic \\ - mutation
}

Neurofibromatosis type 1 (NF1) is a common autosomal dominant disorder with an incidence of $\sim 1$ in 4,000 live births. Neurofibromin, the gene product, is ubiquitously expressed at high levels in the nervous system and functions as a tumor suppressor. Haploinsufficiency of neurofibromin through mutation leads to an increased risk of developing benign and malignant tumors in affected individuals. Although NF1 has complete penetrance, it displays considerable inter- and intrafamilial variability in phenotypic expression which poses disease prediction and management problems. Some NF1 genotype-phenotype correlations have been described. To evaluate the genetic component of variable expressivity in NF1, we examined the phenotypic correlations between affected relatives in 52 NF1 patients from 45 families.

\section{Introduction}

\section{General Remarks}

Neurofibromatosis type 1 (NF1) (MIM \#162200), ${ }^{1}$ also known as von Recklinghausen disease, is a common familial cancer syndrome with a prevalence of 1 in 3000 to 4000 people worldwide, regardless of ethnic and racial background. ${ }^{2}$ NF1 is a dominantly inherited genetic disorder that results from a germline mutation in the NF1 tumor-suppressor gene located on chromosome 17q11.2. The mRNA of the NF1 gene has more than 11 to $13 \mathrm{~kb}$ and encodes a cytoplasmic protein called neurofibromin (NM_000267). ${ }^{3}$ This large (2.818 amino acids, 57 exons) protein is present at low levels in most tissues, with the highest concentration found in the central nervous system (CNS). ${ }^{4}$ Different domains of neurofibromin have been described ( - Fig. 1), but some functions need still to be established. ${ }^{5,6}$ Neurofibromin is essential for normal embryonic development in mammals; the lack of this protein in murine models is lethal mainly because of the altered development of blood vessels. ${ }^{7}$ Neurofibromin functions primarily as a GTPase activating protein (GAP) and inhibits RAS (rat sarcoma protein) activity. As a proto-oncogene, RAS promotes cell proliferation, differentiation, survival, and death. In NF1associated tumors, loss of neurofibromin expression is associated with high levels of active RAS. Depending on the cell

received

October 17, 2017

accepted after revision

December 7, 2017 type, RAS hyperactivation leads to increased signaling through the RAS downstream pathway intermediates, AKT (RAC-alpha serine/threonine-protein kinase)/mTOR (mammalian Target Of Rapamycin) and RAF (rapidly accelerated fibrosarcoma)/ MEK (mitogen-activated protein kinase kinase). This cellular dysregulation explains much of the NF1 clinical phenotype, especially the increased risk of benign and malignant neoplasms evident in NF1.

\section{Phenotype}

Formal clinical criteria for NF1 were published by the National Institutes of Health (NIH) in 1988, and reaffirmed in 1997 (-Table 1). The main features of NF1 include multiple café-au-lait (CAL) spots, Lisch nodules of the iris, axillary freckling, and benign peripheral nerve sheath tumors named neurofibromas. Other NF1-related clinical complications include neurological, renal, cardiovascular, gastrointestinal, endocrine and orthopedic features, learning and intellectual disabilities, attention deficit, and malignancies of the peripheral nerve sheath or CNS. About a quarter of people with NF1 develop one or more of these clinical complications, which cause significant morbidity and mortality. ${ }^{8}$

The incidence of different symptoms is variable and evolves over time with a complete penetrance at 8 years old. CAL spots occur in at least $95 \%$ of patients, whereas other (c) Georg Thieme Verlag KG Stuttgart · New York
DOI https://doi.org/ 10.1055/s-0037-1620239. ISSN $0174-304 X$. 


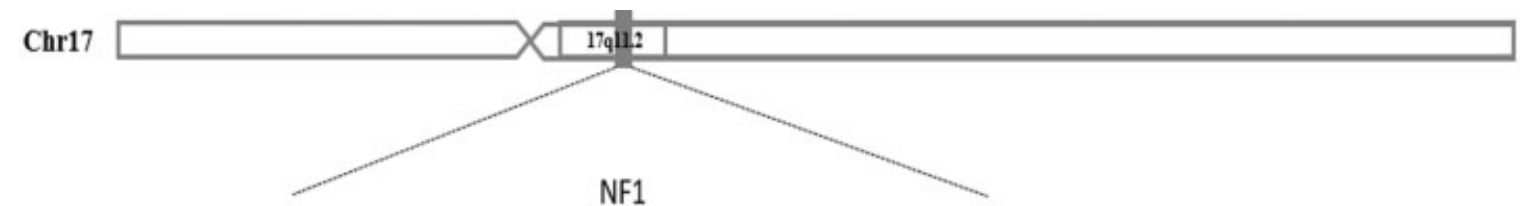

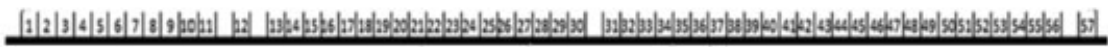

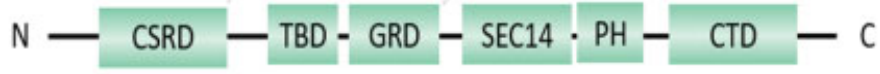

Neurofibromin

Fig. 1 The schema of neurofibromatosis type 1 (NF1) gene and neurofibromin protein. From the upper part: the localization of NF1 gene in chromosome 17; the scheme of NF1 gene with all coding exons of NM_000267 NF1 transcript; the scheme of functional domains of neurofibromin. CSRD, cysteine and serine rich domain; TBD, tubulin binding domain; GRD, domain responsible for interaction with RAS and GTP hydrolysis; Sec14-PH, bipartite lipid binging domain; CTD, carboxyl-terminal domain.

Table 1 Schema of neurofibromin and gene segmentation into six subgroups corresponding to the different functional domains of the protein

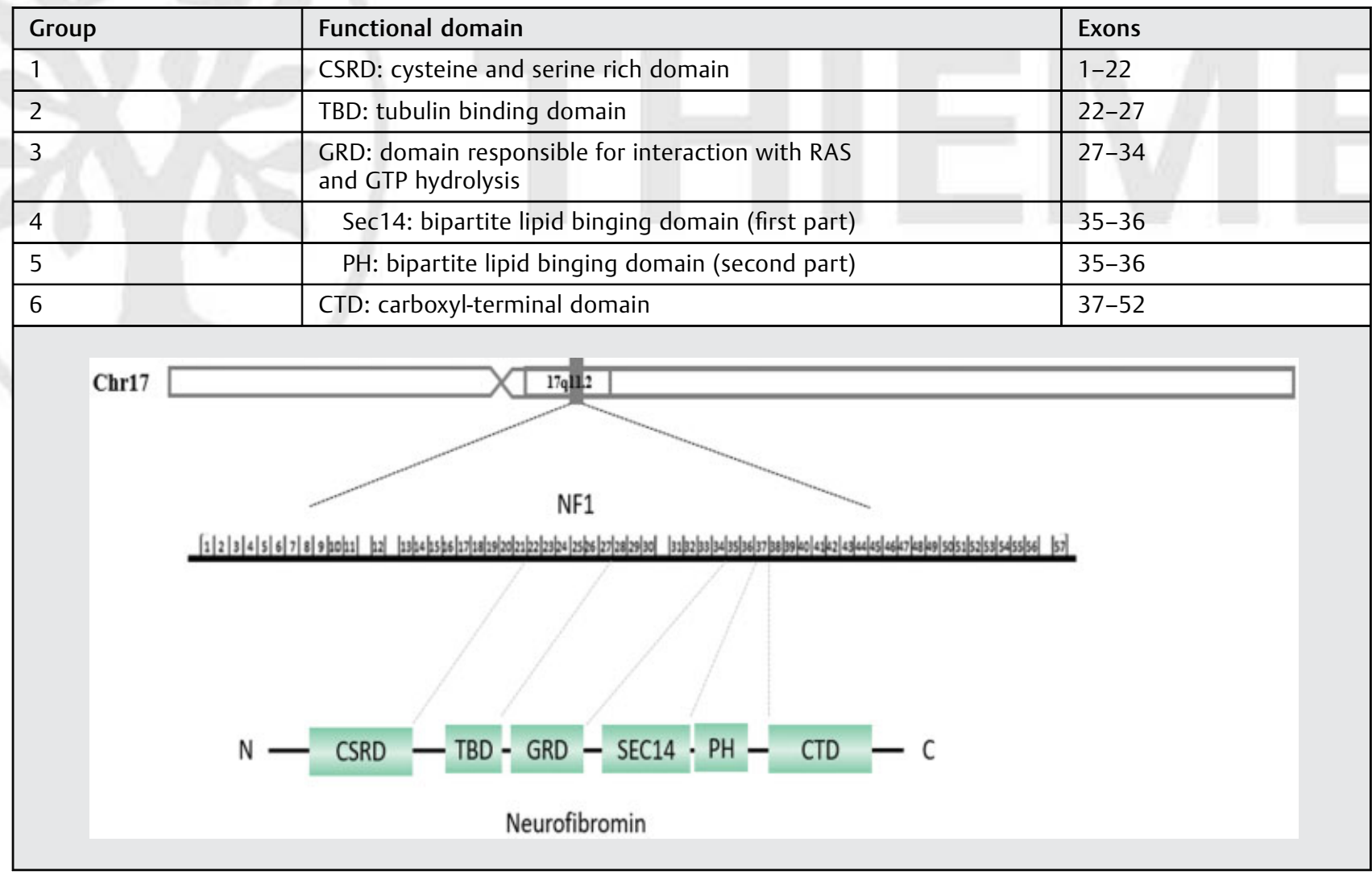

features occur in less than $1 \%$. Otherwise, CAL spots, pseudarthrosis, optic gliomas, and externally visible plexiform neurofibromas are apparent within the first years of life, whereas freckling and severe scoliosis occur from 7 years of age onwards. Subcutaneous neurofibromas and iris Lisch nodules tend to appear in teenage or young adult years
(-Fig. 2). Malignancies are mostly problems of adults. Finally, NF1 is known to be an extraordinarily variable intraand interfamilial condition; some patients have very mild manifestations, whereas others are severely affected, and there is no consistency in manifestations within a sexe or a pedigree. 


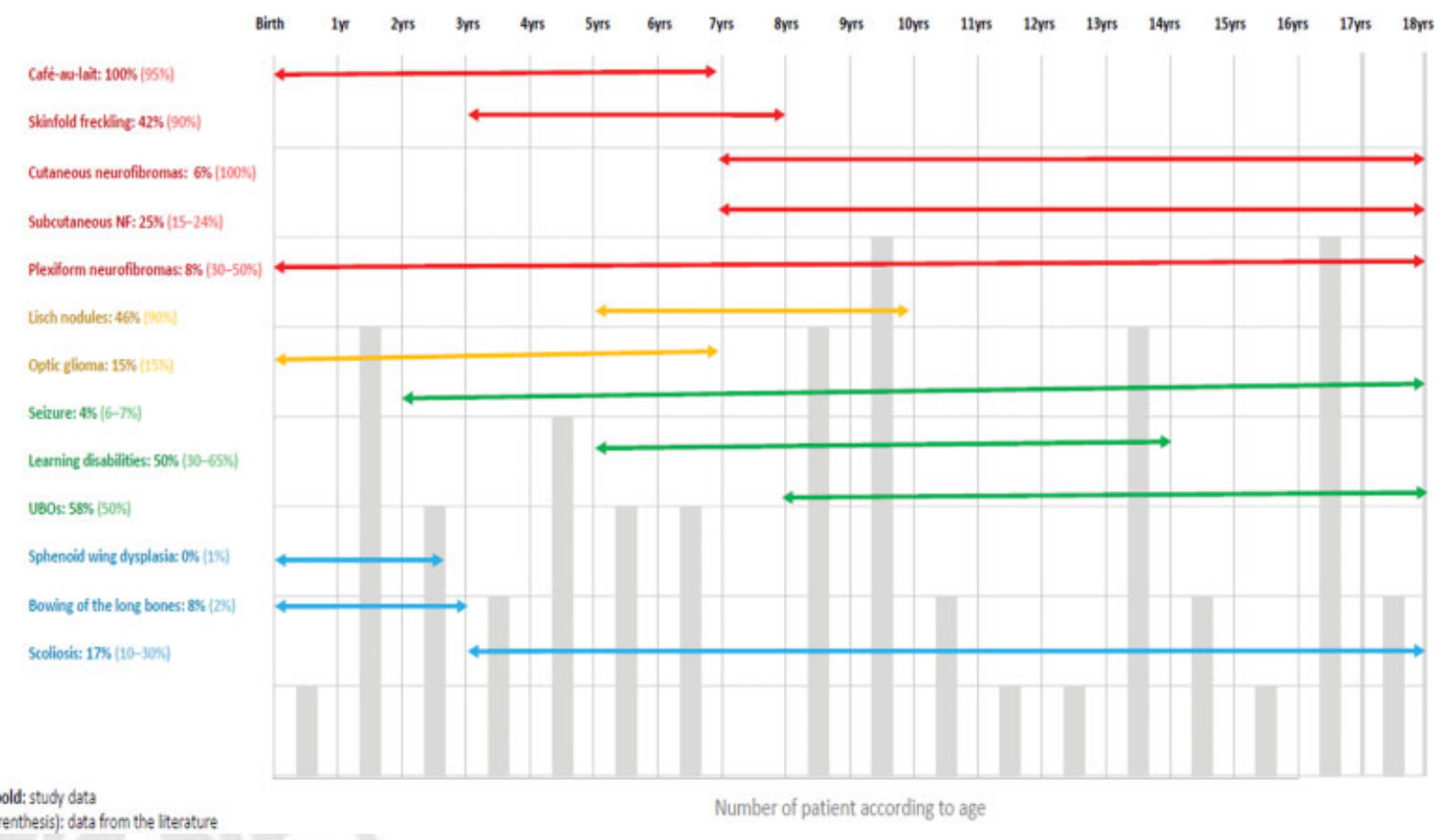

Fig. 2 Neurofibromatosis type 1 (NF1) time line. This figure illustrates at what ages different disease manifestations become apparent. Phenotypic data collection and comparison with literature (in parenthesis) on the x-axis. On the $y$-axis, heterogeneous distribution of the age of the individual patients to be taken into account in the statistical calculations given the age-dependent prevalence of certain symptoms.

Thus, while NF1 represents a simply determined Mendelian disorder with complete penetrance, it is, however, characterized by highly variable expressivity in both the number of major features and the occurrence of complications.

\section{Genotype}

In most cases, the diagnosis of NF1 can be made on clinical grounds; however, genetic testing is necessary to provide prenatal diagnosis and may be used as an adjunct to clinical diagnosis in cases with an atypical presentation or in which the child is too young to have developed most characteristic features. When employed, NF1 mutation analysis is $95 \%$ sensitive. ${ }^{9}$ About $50 \%$ of NF1 patients are de novo mutations. Ninety percent of de novo mutations occur on a paternal chromosome, while large deletions were of maternal origin. ${ }^{10}$ The incidence of de novo mutations in NF1 gene locus is very high compared with other genes involved in the pathogenesis of genetically determined diseases. The large size of NF1 gene does not fully explain such a high rate of new mutation formation. Mutations identified so far are randomly distributed within the gene, and they are not localized in "hot spot" regions which are more prone to the mutation occurrence. At least more than a thousand different NF1 gene mutations have been identified, ${ }^{11}$ many being unique to a specific individual or family; less than $20 \%$ of NF1 gene lesions are recurrent. Based on the Human Gene Mutation Database (HGMD) database (august 2017), of the 1690 mutations described, most of these DNA changes are small deletions (26.1\%), missense/nonsense (23.8\%), and splicing mutations (20.9\%). With the advent of accurate genetic testing, early genotype-phenotype correlations are begin- ning to emerge: People with genomic microdeletions affecting the entire NF1 gene have a more severe phenotype; this contiguous gene syndrome does appear to include dysmorphic features, learning disabilities, cardiovascular malformations, childhood overgrowth, a higher tumor burden and earlier onset of benign neurofibromas, and probably a higher incidence of malignancies. ${ }^{12}$ By comparison with patients harboring other mutation types, NF1 patients with splice-site mutations were found to have an increased number of subcutaneous neurofibromas, a tendency to intracerebral gliomas, malignant peripheral nerve sheath tumors, and more preschool learning difficulties. ${ }^{13}$ Individuals with missense mutations have less Lisch nodules. ${ }^{14}$ Patients with NF1 with the specific c.2970-2972delAAT mutation do not develop cutaneous, subcutaneous, and superficial plexiform neurofibromas. ${ }^{5}$

\section{Hypothesis}

As neurofibromin appears everywhere, NF1 is a complex disorder that affects many cell types and involves multiple body systems. However, NF1 is noted for the considerable inter and intrafamilial variation observed in the clinical phenotype even in patients who share the same germline mutation. As a result, any decisions on surveillance tailored to some of these potentially treatable complications can be difficult to implement in the clinic. Successful genetic counseling is also hampered by clinical complications that are unpredictable both in severity and progression even within the same family.

Given the clinical feeling of a more pronounced symptomatology in some families, and the description of some correlates in the literature, ${ }^{15}$ the research for a phenotype- 
genotype correlation remains legitimate. The present retrospective study attempts to assess whether any associated genotype-phenotype correlations could be identified in a cohort of clinically well-characterized NF1 patients with known pathogenic NF1 mutations.

\section{Materials and Methods}

\section{Patients}

The present study is a retrospective analysis of the clinical data obtained from a cohort of NF1 patients collected between the 1st January 2014 and the 31 December 2016 in the Centre Hospitalier Régional (CHR) of Liege, Belgium. The study cohort comprised 52 NF1 germline mutation-positive patients who are followed by a multidisciplinary team responsible for the diagnosis and the management of NF1 in children from 0 to 18 years.

The patient cohort was characterized by a majority of boys (sex ratio boy/girl $=3 / 2$ ), an ethnic origin that was predominantly Caucasian, and an age distribution which ranged from 0 to 18 (mean age: 9.7 years). Twenty-two patients have a positive family history of NF1, 28 patients are sporadic, and 2 have an unknown family history.

\section{Clinical Features and Associated Mutations}

Major NF1 clinical features were recorded for each patient, alongside patient gender, the inherited or noninherited character, and the type of NF1 germline mutations. These mutations are also recorded into six subgroups corresponding to the different functional domains of the protein ( - Table 2 ).

The recorded clinical details included the presence of CAL spots, subcutaneous neurofibromas, plexiform neurofibromas, freckling, Lisch nodules of the iris, optic gliomas, seizures, evidence of preschool learning difficulties, unidentified bright objects (UBOs) which are T2 hyperintense foci on MRI, long bone dysplasias, and scoliosis of more than $10^{\circ}$. No other tumor known to be commonly associated with NF1 was recorded.

Table 2 Diagnostic criteria for neurofibromatosis type 1 (NF1) established by the National Institutes of Health consensus development conference (1988)

\begin{tabular}{l} 
Clinical criteria of NF1 \\
Two or more of the followings must be present to \\
assess the diagnosis \\
Six or more café-au-lait macules $>0.5 \mathrm{~cm}$ in the \\
prepubertal child or $>1.5 \mathrm{~cm}$ after puberty \\
\hline Skinfold freckling \\
\hline Two or more neurofibromas of any type ore one plexiform \\
neurofibroma \\
\hline two or more Lisch nodules \\
\hline Optic pathway glioma \\
\hline Bony dysplasia \\
\hline A first-degree relative with NF1
\end{tabular}

\section{Statistical Tool}

Logistic regression analysis was performed to test the association of each clinical feature individually with the type of constitutional NF1 mutation. Each regression model had the covariates of age at examination (as a quantitative variable) to control for potential confounding. All statistical analyses were performed with XLSTAT software (Addinsoft, New York, United States).

\section{Results}

The identified constitutional NF1 gene alterations appear to be uniformly distributed across the gene. The prevalence of these common clinical abnormalities in this study was similar to those of other published studies (-Fig. 2 ).

The results of this analysis revealed no significant correlation between each phenotypical presentation and the mutations, whether grouped according to their type or functional domains (1-6). Moreover, gender and parental imprinting have no effect on phenotypic presentation in our study.

\section{Discussion}

\section{Correlation}

We did not find any genotype-phenotype correlation for common clinical features of NF1, similar to the findings of most other studies. Owing to the absence of strong genotype-phenotype correlations, NF1 mutation analysis has for now very limited predictive utility for specific sequelae. However, it has greater diagnostic utility because mutation detection can confirm the etiology of the disease in individuals in whom the clinical phenotype does not fulfill the NIH diagnostic criteria. A firm diagnosis of NF1 directs the patient toward a multidisciplinary management with a specific monitoring. It allows counseling regarding mode of inheritance, recurrence risk, and potentially prenatal diagnosis too.

The limitations of this study include the restricted number of patients, the low number of patients with the same mutation, the low number of genetic deletions, and the low representation of some phenotypes. As the penetrance of many NF1 disease features increases with age, there is a potential bias toward lower age at examination in nonprobands relative to probands. Nevertheless proband status did not contribute significantly to any of the models after including age at examination as a covariate. Finally, it should be noted that the CHR of Liège is a regional center of expertise which drains only a part of the sick population, which seems therefore not representative of the general population.

\section{Atypical Cases}

One patient has c.4030G > T NF1 mutation associated with JeffCampanaccy syndrome. This syndrome describes the complex of multiple nonossifying fibromas (NOFs) of the long bones, giant cell granulomas (GCG) of the jaw, and CAL macules. It has been demonstrated that the majority of patients with these symptoms harbored a pathogenic germline NF1 mutation, ${ }^{16}$ and that inactivation of the NF1 gene may contribute to the development of GCG, ${ }^{17}$ suggesting that many Jaffe-Campanacci 
syndrome cases may actually have NF1. In this context, giant cell lesions seem to be NF1-associated tumors.

Two of our NF1 patients, brothers and sisters, are both carriers of a neomutation. Interestingly, the two mutations found are not the same. The first child, a 14-year-old girl with a mosaic deletion of exons 10 to 15 , presents CAL spots, frecklings, a subcutaneous neurofibroma, Lisch nodules, mild scoliosis, UBO, and a glioma of the optical pathways. The second child, a 10 -year-old boy with a missense c. $4768 \mathrm{C}>$ T mutation, presents only CAL spots.

As we know, neurofibromin has an important impact on the vascular and orthopedic development. We described here two cases with atypical presentations not described in the literature. The first case is a boy with NF1 mutation c.1642-2A > G who developed a hemihypertrophia of the right foot associated with a vascular malformation. This presentation was not found in his two brothers and in his mother who carry the same mutation. The second case concerns a boy with a c.2410-16 A-G mutation who developed tibial amyotrophy, not present in his sister and in his father who carry the same mutation.

\section{Conclusion}

In our study, the mutation spectrum of the NF1 gene is similar to previously reported, confirming the absence of hot spot regions and obvious genotype-phenotype correlations.

Despite its limited predictive utility, genetic analysis is interesting for early diagnosis and in the case of atypical presentation of the disease. Finally, this study reports different atypical presentation.

Although this pathology is well recognized and described for several years, there remain uncertainties about the genetic and physiological pathways that require more investigations.

\section{Conflict of Interest \\ None.}

\section{References}

1 Online Mendelian Inheritance in Man Database - Entry \#162200. http://www.omim.org/entry/162200. Accessed January 1, 2017
2 Anderson JL, Gutmann DH. Neurofibromatosis type 1. Handb Clin Neurol 2015;132:75-86

3 Hirbe AC, Gutmann DH. Neurofibromatosis type 1: a multidisciplinary approach to care. Lancet Neurol 2014;13(08):834-843

4 Marchuk DA, Saulino AM, Tavakkol R, et al. cDNA cloning of the type 1 neurofibromatosis gene: complete sequence of the NF1 gene product. Genomics 1991;11(04):931-940

5 Upadhyaya M, Huson SM, Davies M, et al. An absence of cutaneous neurofibromas associated with a 3-bp inframe deletion in exon 17 of the NF1 gene (c.2970-2972 delAAT): evidence of a clinically significant NF1 genotype-phenotype correlation. Am J Hum Genet 2007;80(01):140-151

6 Abramowicz A, Gos M. Neurofibromin in neurofibromatosis type 1 - mutations in NF1gene as a cause of disease. Dev Period Med 2014;18(03):297-306

7 Trovó-Marqui AB, Tajara EH. Neurofibromin: a general outlook. Clin Genet 2006;70(01):1-13

8 Upadhyaya M. Neurofibromatosis type 1: diagnosis and recent advances. Expert Opin Med Diagn 2010;4(04):307-322

9 Valero MC, Martín Y, Hernández-Imaz E, et al. A highly sensitive genetic protocol to detect NF1 mutations. J Mol Diagn 2011;13 (02):113-122

10 Ben-Salem S, Al-Shamsi AM, Ali BR, Al-Gazali L. The mutational spectrum of the NF1 gene in neurofibromatosis type I patients from UAE. Childs Nerv Syst 2014;30(07):1183-1189

11 The Human Gene Mutation Databasehttp:/www.hgmd.org. Accessed January 1, 2017

12 Pasmant E, Sabbagh A, Spurlock G, et al; members of the NF France Network. NF1 microdeletions in neurofibromatosis type 1: from genotype to phenotype. Hum Mutat 2010;31(06):E1506-E1518

13 Alkindy A, Chuzhanova N, Kini U, Cooper DN, Upadhyaya M. Genotype-phenotype associations in neurofibromatosis type 1 (NF1): an increased risk of tumor complications in patients with NF1 splice-site mutations? Hum Genomics 2012;6:12

14 Castle B, Baser ME, Huson SM, Cooper DN, Upadhyaya M. Evaluation of genotype-phenotype correlations in neurofibromatosis type 1. J Med Genet 2003;40(10):e109

15 Sabbagh A, Pasmant E, Laurendeau I, et al; members of the NF France Network. Unravelling the genetic basis of variable clinical expression in neurofibromatosis 1. Hum Mol Genet 2009;18(15):2768-2778

16 Stewart DR, Brems H, Gomes AG, et al. Jaffe-Campanacci syndrome, revisited: detailed clinical and molecular analyses determine whether patients have neurofibromatosis type 1 , coincidental manifestations, or a distinct disorder. Genet Med 2014;16(06):448-459

17 Friedrich RE, Mautner VF, Scheuer HA. Loss of heterozygosity in tumor cells of a recurrent mandibular giant cell granuloma in neurofibromatosis type 1. Anticancer Res 2007;27(4A):2079-2083 